\title{
No association between myeloperoxidase gene rs2333227 G>A polymorphism and Alzheimer's disease risk: a meta-analysis and trial sequential analysis
}

\author{
Zhi-Cheng Fang ${ }^{1,2, \dagger}$, Chen-Chen $\mathrm{Mao}^{3, \dagger}$, Ya-Jun Hu${ }^{3}$, Gong-Li Yang ${ }^{2,4, *}$, Lan Zhou ${ }^{1,2, *}$ \\ ${ }^{1}$ Department of Emergency, Taihe Hospital, Hubei Key Laboratory of Embryonic Stem Cell Research, Hubei University of Medicine, 442000 Shiyan, Hubei, \\ China \\ ${ }^{2}$ Department of Neurology, Taihe Hospital, Hubei University of Medicine, 442000 Shiyan, Hubei, China \\ ${ }^{3}$ Department of Stomatology \& Center for Evidence-Based Medicine and Clinical Research, Taihe Hospital, Hubei Key Laboratory of Embryonic Stem Cell \\ Research, Hubei University of Medicine, 442000 Shiyan, Hubei, China \\ ${ }^{4}$ Department of Gastroenterology, Shenzhen University General Hospital, Shenzhen University, 518060 Shenzhen, Guangdong, China \\ *Correspondence: glyang169@gmail.com (Cong-Li Yang); zhoulan123lm@gmail.com (Lan Zhou) \\ ${ }^{\dagger}$ These authors contributed equally.
}

DOI:10.31083/j.jin2101032

This is an open access article under the CC BY 4.0 license (https://creativecommons.org/licenses/by/4.0/).

Submitted: 7 April 2021 Revised: 7 May 2021 Accepted: 9 August 2021 Published: 28 January 2022

Evidence suggests that there is a close association between myeloperoxidase (MPO) gene rs2333227 G > A polymorphism with Alzheimer's disease (AD) susceptibility. We conducted a metaanalysis to explore the precise association between MPO rs2333227 $\mathrm{G}>\mathrm{A}$ polymorphism and AD susceptibility. Online databases were searched and the relevant information was collected. Crudeodds ratios with $95 \%$ confidence intervals were calculated. Trial sequential analysis (TSA), heterogeneity analyses, accumulative analyses, sensitivity analyses, and publication biasestests were performed. Overall, nine publications (ten independent case-controls) were included in this meta-analysis, involving 3260 participants. Pooled results revealed no significant association between MPO rs2333227 $\mathrm{G}>\mathrm{A}$ polymorphism and AD susceptibility was observed. TSA showed that the present meta-analysis remained inconclusive due to insufficient evidence. In summary, the current meta-analysis indicated that the MPO rs2333227 G > A polymorphism may not be acausalfactor in the development of $A D$.

\section{Keywords}

Alzheimer's disease; Myeloperoxidase; Polymorphism; Trial sequential analysis

\section{Introduction}

Alzheimer's disease (AD) is a chronic, progressive and irreversible neurodegenerative disease. The prevalence of $\mathrm{AD}$ is continuously rising due to the increasing longevity of the global population [1]. AD is the most common cause of dementia and is considered one of the largest health challenges worldwide. According to a recent epidemiological report, 4.7 million individuals aged 65 years or older are estimated to have $\mathrm{AD}$ dementia, and the disease will cause more than 600,000 deaths, comprising $32 \%$ of all older adult deaths in the US. By 2050, the number of people with AD dementia is projected to be 13.8 million, causing approximately 1.6 million deaths, thereby accounting for $43 \%$ of all older adult deaths $[2,3]$. AD cases are likely to increase among developing and developed countries, and their complications will place tremendous mental and economic burdens on patients and families. Globally, AD and other dementias are considered to be one of the most important causes of disability adjusted life years (DALYs), accounting for 28.8 million DALY $\sin 2016$ [4].

To date, the etiology and pathogenesis of $\mathrm{AD}$ have been extensively explored, but still, the underlying mechanisms remain unclear. Increasing evidence suggests that the accumulation of abnormally folded amyloid $\beta$ protein $(\mathrm{A} \beta)$ and tau proteins in amyloid plaques and neuronal tangles are causally related to neurodegenerative processes in $\mathrm{AD}$ patients $[5,6]$. Other factors such as cerebrovascular pathological changes, diabetes, chronic inflammation [7], and unhealthy life styles have also been proposed to be associated with AD development. Moreover, increasing evidence suggests that genetic abnormalities, including genetic mutations, may confer an increased risk of $\mathrm{AD}$ [8]. The mutation of apolipoprotein $\mathrm{E}(\mathrm{ApoE}) \epsilon 4$ is a critical genetic risk factor for $\mathrm{AD}$, and the risk of $\mathrm{AD}$ lifetime risks rose to $51 \%$ for ApoE $\epsilon 4 / \epsilon 4$ male carriers to $60 \%$ for ApoE $\epsilon 4 / \epsilon 4$ female carriers, as well as for ApoE $\epsilon 3 / \epsilon 4$ carriers [9]. Other mutations in amyloid precursor protein genes can influence the accumulation and clearance of $\mathrm{A} \beta$ and cause $\mathrm{AD}$ [10]. Moreover, many genetic loci have been identified to associate with AD risk by genomewide association studies (GWAS) [11, 12].

Myeloperoxidase (MPO) is an oxidative lysosomal enzyme released by activated polymerphonuclear neutrophils. MPO participates in the defense mechanisms of an organism through its catalytic activities in the hydrogen peroxide- 
halide reaction to kill infectious pathogens [13]. Studies have reported that chronic inflammation with oxidative stress contributes to the development of numerous neurodegenerative conditions, such as $\mathrm{AD}$, Parkinson's disease, multiple sclerosis and stroke. Epidemiological studies have demonstrated a positive association between aberrant MPO plasma levels, and AD and cardiovascular disease susceptibility [14]. The MPO gene is located on the long arm of chromosome $17 \mathrm{q} 23.1$ in humans, is $14 \mathrm{~kb}$ in length, and comprises $12 \mathrm{ex}-$ ons and 11 introns [15]. To date, many single nucleotide polymorphisms (SNPs) loci have been identified in the MPO gene and the rs2333227 G>A (-463 G>A) polymorphism was first identified in the promoter region by Austin et al. [16] in leukemic bone marrow cells. The MPO A allele disrupts the Sp1transcription factor binding region and affects transcription; carriers show decreased mRNA expression and transcriptional activity compared with the wild-type $G$ allele carriers [17].

Since 1999, several case-control studies have been conducted to investigate the role of this polymorphism and $\mathrm{AD}$ susceptibility, but their results were inconsistent. In 2002, Combarros et al. [18] conducted the first meta-analysis on the association between MPO rs2333227 G>A polymorphism and $\mathrm{AD}$ risk. Regrettably, only four case-control studies were conducted, and the hypothesis that MPO rs2333227 G>A polymorphism was causally related to $\mathrm{AD}$ was nullified. Considering the present situation and the absence of a comprehensive evaluation, this meta-analysis was conducted to more accurately assess the association between MPO rs2333227 $\mathrm{G}>\mathrm{A}$ polymorphism, and $\mathrm{AD}$ risk.

\section{Materials and methods}

This current meta-analysis was conducted with the guidance of the Preferred Reporting Items for Systematic Reviews and Meta-Analyses (PRISMA) statement. All collected data were extracted from published studies; hence, there were no ethics issues to consider.

\subsection{Searching strategy}

Online databases (PubMed, Embase, Web of Science, CNKI and Wanfang) were searched for all published casecontrol studies on the association between MPO rs2333227 $\mathrm{G}>\mathrm{A}$ polymorphism and $\mathrm{AD}$ susceptibility from inception up to December 1, 2020. Only articles published in English or Chinese were selected. The bibliographies of all identified studies were reviewed for further potential studies of interest. The following search terms were used: "(myeloperoxidase OR MPO) AND (-463 OR rs2333227 OR polymorphism OR variant OR mutation) AND ((Alzheimer's disease) OR dementia)", and the strategy was listed (e.g., in PubMed):

\#1 myeloperoxidase

\#2 MPO

\#3 \#1 OR \#2

$\# 4-463$

\#5 rs2333227

\#6 polymorphism
\#7 variant

\#8 mutation

\#9 \#4 OR \#5 OR \#6 OR \#7 OR \#8

\#10 Alzheimer's disease

\#11 dementia

\#12\#10 OR \#11

\#15 \#3 AND \#9 AND \#12

\subsection{Inclusion and exclusion criteria}

Studies were included if they were: (1) case-control studies that examined the association between MPO rs2333227 G>A polymorphism and AD susceptibility; (2) studies that assessed genotypes related information of the genotypes distributions in the case and control groups to determine odds ratios (ORs) and 95\% confidence intervals (CIs); and (3) studies that were published only in English. In cases where there was an overlap in data reported by different studies, we used the study that contained the largest or most recent data. We excluded: (1) case reports, review articles or animal studies; (2) molecular biology research not addressing the relationship between MPO rs2333227 G > A polymorphism, and AD risk; (3) studies without sufficient data; and (4) studies with duplicated or overlapping data.

\subsection{Data extraction and quality evaluation}

Two authors (Fang and Mao) reviewed the included studies independently, and the following information was extracted and listed for analysis: the first authors' name, publication date, study country, sample sizes of the cases and controls, control design, genotyping method, frequency data of the genotype distribution, and assessment of hardy-weinberg equilibrium (HWE) in the control group. The newcastleottawa scale (NOS) was employed to evaluate the quality of all included studies. The scores ranged from 0 (worst) to 9 (best). Studies with a score of 7 points or higher indicated that they were of good research quality [19].

\subsection{Statistical analysis}

Overall, pooled ORs and 95\% CIs were calculated to evaluate the association between MPO rs2333227 G>A polymorphism and AD susceptibility. Heterogeneity among the included studies was examine dusing Cochran's Q test and $\mathrm{I}^{2}$ test. $\mathrm{I}^{2}>40 \%$ or $P<0.1$ was considered indicative of significant heterogeneity. The fixed-effect model (MantelHaenszel method) was adopted where heterogeneity was not significant; otherwise, a random-effects model (I-V heterogeneity method model) was adopted. Genetic models were applied, including the allele contrast model (A vs. G), the codominant model (heterozygote comparison: GA vs. GG and homozygote comparison: AA vs. GG), the dominant model (GA + AA vs. GG), and the recessive model (AA vs. GG + GA). Subgroup analyses were conducted based on HWE status, ethnicity differences, control design, and NOS evaluation. Meta-regression was performed to explore the potential factors contributing to heterogeneity. Cumulative metaanalyses and sensitivity analyses were conducted to examine the statistical tendency and stability of the results. Egger's 


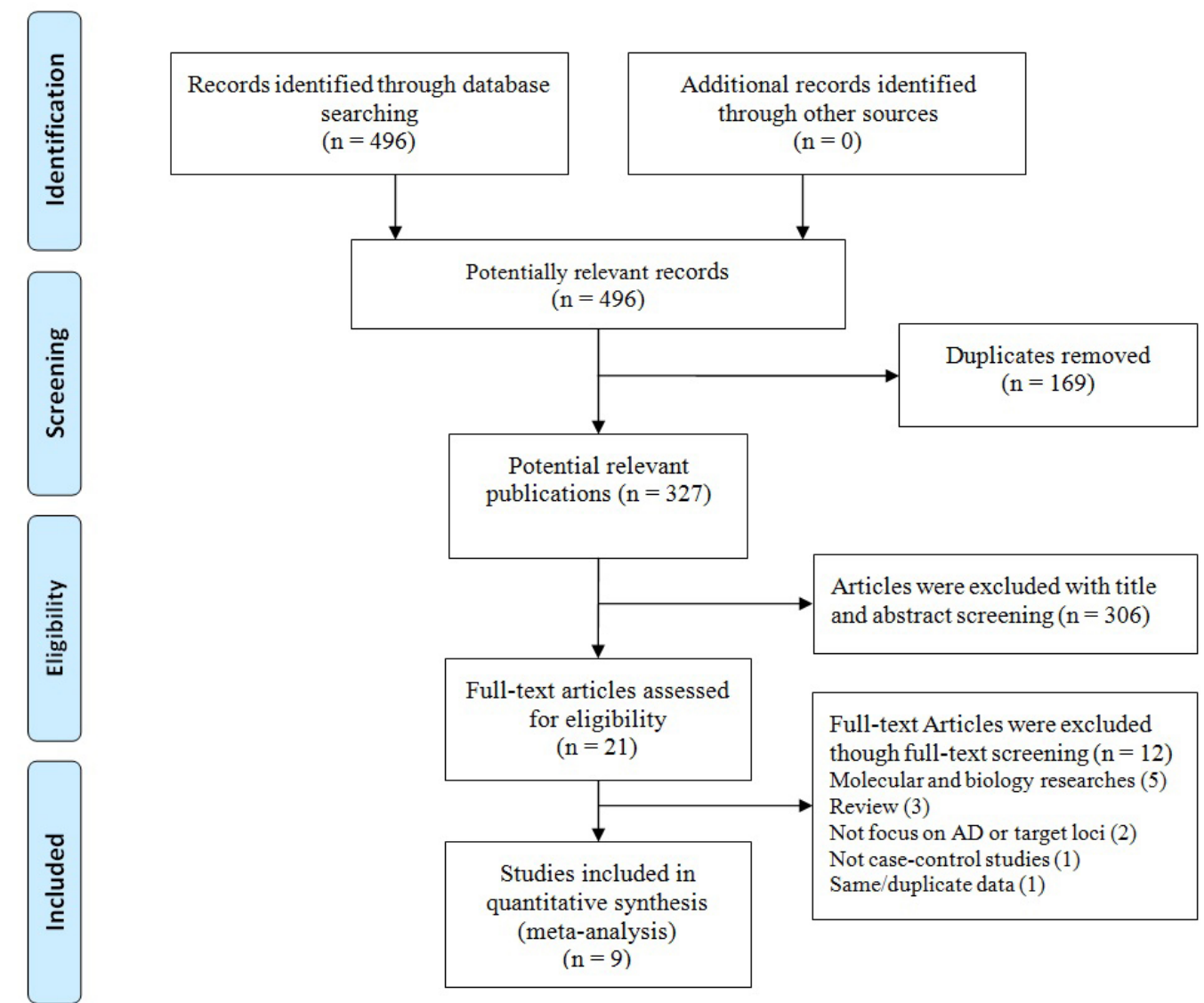

Fig. 1. Flow diagram of the study selection process.

[20] linear regression test was applied and Begg's [21] funnel plots were constructed to explore whether there was publication bias. Finally, a trial sequential analysis (TSA) was conducted [22]. All the statistical calculations were conducted using Stata version 14.0 (Stata Corporation, College Station, TX, USA). A two-sided $P$-value $<0.05$ was considered statistically significant.

\section{Results}

\subsection{Study characteristics}

The selection process is presented in Fig. 1. In the first step of the literature search, 496 potential case-control studies were identified. A total of 169 studies were excluded due to duplication, 306 studies were deleted through fulltext review or title and abstract screening, and 12 studies were excluded for other deficiencies. Then, nine publications (10 independent case-control studies) involving 1630 patients and 1630 controls were included to assess the association between MPO rs2333227 G > A polymorphism with $\mathrm{AD}$ risk [18, 23-30]. Eight studies were conducted in Caucasian or European populations, and two studies were conducted in Asian populations. All the studies used the polymerase chain reaction-restriction fragment length polymorphism (PCR-RFLP) genotyping method. In terms of genotype distributions, only two studies had control groups that deviated from HWE (Table 1). Overall, the studies included in this work were of moderate to high quality according to the NOS evaluation (Table 1).

\subsection{Quantitative analyses of MPO rs2333227 G>A polymorphism and $A D$ susceptibility}

Overall, the pooled results did not show any significant association between MPO rs2333227 G > A polymorphism and AD susceptibility under any genetic model (A vs. G: OR = $1.09,95 \% \mathrm{CI}=0.85-1.40, P=0.51, \mathrm{I}^{2}=73.9 \%$; GA vs. GG: $\mathrm{OR}=1.04,95 \% \mathrm{CI}=0.83-1.31, P=0.75, \mathrm{I}^{2}=47.5 \%$; AA vs. GG: $\mathrm{OR}=1.16,95 \% \mathrm{CI}=0.61-2.19, P=0.65, \mathrm{I}^{2}=68.5 \%$; GA + AA vs. GG: $\mathrm{OR}=1.06,95 \% \mathrm{CI}=0.83-1.34, P=0.65, \mathrm{I}^{2}=$ $59.3 \%$ (Fig. 2); AA vs. GG + GA: OR $=1.14,95 \% \mathrm{CI}=0.64$ 2.02, $P=0.65, \mathrm{I}^{2}=62.3 \%$ ) (Table 2 ). In the stratified analysis, negative associations were found between MPO rs2333227 $\mathrm{G}>\mathrm{A}$ polymorphism and AD susceptibility based on HWE status, ethnicity diversity, control design, and NOS evaluation.

\subsection{Heterogeneity and publication bias}

Initially, some heterogeneity was observed among the included studies in the general population under all five genetic models (A vs. G: $\mathrm{I}^{2}=73.9 \%$; GA vs. GG: $\mathrm{I}^{2}=47.5 \%$; AA vs. GG: $\mathrm{I}^{2}=68.5 \%$; GA + AA vs. GG: $\mathrm{I}^{2}=59.3 \%$; AA vs. GG + GA: $\left.\mathrm{I}^{2}=62.3 \%\right)$. Many factors, such as HWE status, ethnicity 
Table 1. Characteristics of case-control studies on MPO rs2333227 G >A polymorphism and Alzheimer's disease risk included in the meta-analysis.

\begin{tabular}{|c|c|c|c|c|c|c|c|c|c|c|c|c|c|c|c|}
\hline \multirow{3}{*}{ First author } & \multirow{3}{*}{ Year } & \multirow{3}{*}{ Country/Ethnicity } & \multirow{3}{*}{ Control design } & \multirow{3}{*}{ Case } & \multirow{3}{*}{ Control } & \multicolumn{6}{|c|}{ Genotype distribution } & \multirow{3}{*}{$P$ for HWE } & \multirow{3}{*}{ Genotyping method } & \multirow{3}{*}{ MAF } & \multirow{3}{*}{ NOS } \\
\hline & & & & & & \multicolumn{3}{|c|}{ Case } & \multicolumn{3}{|c|}{ Control } & & & & \\
\hline & & & & & & GG & GA & AA & GG & GA & AA & & & & \\
\hline Reynolds-1 & 1999 & US & Not available & 69 & 160 & 43 & 25 & 1 & $96^{a}$ & $64^{b}$ & NA & NA & PCR-RFLP & NA & 5 \\
\hline Reynolds-2 & 2000 & Finnish & Hospital-based & 127 & 174 & 77 & 45 & 5 & 117 & 54 & 3 & 0.25 & PCR-RFLP & 0.17 & 7 \\
\hline Crawford-A & 2001 & US & Population-based & 226 & 166 & 138 & 70 & 18 & 83 & 62 & 21 & 0.09 & PCR-RFLP & 0.31 & 8 \\
\hline Crawford-B & 2001 & US & Population-based & 59 & 75 & 26 & 27 & 6 & 42 & 29 & 4 & 0.73 & PCR-RFLP & 0.25 & 8 \\
\hline Combarros & 2002 & Spain & Hospital-based & 315 & 327 & 160 & 142 & 13 & 168 & 139 & 20 & 0.21 & PCR-RFLP & 0.27 & 7 \\
\hline Styczynska & 2003 & Poland & Not available & 100 & 100 & 71 & 10 & 19 & 72 & 17 & 11 & $<0.01$ & PCR-RFLP & 0.20 & 6 \\
\hline Leininger-Muller & 2003 & Europe & Hospital-based & 265 & 246 & 147 & 101 & 17 & 157 & 84 & 5 & 0.10 & PCR-RFLP & 0.19 & 6 \\
\hline Zappia & 2004 & Italy & Healthy-based & 148 & 158 & 84 & 60 & 4 & 67 & 74 & 17 & 0.61 & PCR-RFLP & 0.34 & 7 \\
\hline Usui & 2006 & Japan & Population-based & 205 & 92 & 166 & 38 & 1 & 75 & 15 & 0 & 0.39 & PCR-RFLP & 0.08 & 8 \\
\hline $\mathrm{Ji}$ & 2017 & China & Hospital-based & 116 & 134 & 68 & 41 & 7 & 95 & 34 & 5 & 0.08 & PCR-RFLP & 0.16 & 7 \\
\hline
\end{tabular}

HWE in control.

${ }^{a}$ Data of the GG genotype; ${ }^{b}$ Data of the GA/AA genotypes.

MAF, Minor allele frequency in control group; PCR-RFLP, Polymerase chain reaction-restriction fragment length polymorphism.

Table 2. Summary ORs and 95\% CI of MPO rs2333227 G > A polymorphism and Alzheimer's disease risk.

\begin{tabular}{|c|c|c|c|c|c|c|c|c|c|c|c|c|c|c|c|c|c|c|c|c|c|}
\hline & \multirow{2}{*}{$\mathrm{N}^{*}$} & \multicolumn{4}{|c|}{ A vs. $G$} & \multicolumn{4}{|c|}{ GA vs. GG } & \multicolumn{4}{|c|}{ AA vs. GG } & \multicolumn{4}{|c|}{ GA + AA vs. GG } & \multicolumn{4}{|c|}{ AA vs. GG + GA } \\
\hline & & OR & $95 \% \mathrm{CI}$ & $P$ & $I^{2}(\%)$ & OR & $95 \% \mathrm{CI}$ & $P$ & $I^{2}(\%)$ & OR & $95 \% \mathrm{CI}$ & $P$ & $I^{2}(\%)$ & OR & $95 \% \mathrm{CI}$ & $P$ & $I^{2}(\%)$ & OR & $95 \% \mathrm{CI}$ & $P$ & $I^{2}(\%)$ \\
\hline Total & 10 & 1.09 & $0.85-1.40$ & 0.51 & 73.9 & 1.04 & $0.83-1.31$ & 0.75 & 47.5 & 1.16 & $0.61-2.19$ & 0.65 & 68.5 & 1.06 & $0.83-1.34$ & 0.65 & 59.3 & 1.14 & $0.64-2.02$ & 0.65 & 62.3 \\
\hline HWE-yes & 8 & 1.07 & $0.81-1.40$ & 0.64 & 76.5 & 1.07 & $0.85-1.35$ & 0.55 & 48.2 & 1.09 & $0.53-2.25$ & 0.81 & 69.9 & 1.08 & $0.81-1.43$ & 0.60 & 68.0 & 1.05 & $0.56-1.98$ & 0.87 & 62.1 \\
\hline \multicolumn{22}{|l|}{ Ethnicity } \\
\hline Caucasian & 8 & 1.03 & $0.77-1.37$ & 0.85 & 77.4 & 0.97 & $0.75-1.25$ & 0.81 & 49.5 & 1.08 & $0.52-2.25$ & 0.83 & 75.0 & 0.99 & $0.76-1.29$ & 0.94 & 61.3 & 1.09 & $0.56-2.11$ & 0.80 & 70.7 \\
\hline Asian & 2 & 1.44 & $1.00-2.06$ & 0.05 & 0 & 1.44 & $0.94-2.19$ & 0.09 & 0 & 1.87 & $0.61-5.71$ & 0.27 & 0 & 1.48 & $0.98-2.23$ & 0.06 & 0 & 1.61 & $0.54-4.87$ & 0.40 & 0 \\
\hline \multicolumn{22}{|l|}{ Design } \\
\hline $\mathrm{HB}$ & 5 & 1.10 & $0.78-1.55$ & 0.59 & 80.3 & 1.12 & $0.85-1.48$ & 0.40 & 51.5 & 1.15 & $0.41-3.19$ & 0.79 & 78.1 & 1.12 & $0.80-1.59$ & 0.51 & 71.5 & 1.10 & $0.44-2.77$ & 0.84 & 73.9 \\
\hline $\mathrm{PB}$ & 3 & 1.02 & $0.59-1.78$ & 0.94 & 73.6 & 0.99 & $0.60-1.66$ & 0.95 & 51.0 & 0.99 & $0.31-3.20$ & 0.98 & 51.7 & 1.01 & $0.56-1.81$ & 0.97 & 67.6 & 0.79 & $0.44-1.40$ & 0.42 & 26.4 \\
\hline NA & 2 & 1.30 & $0.81-2.10$ & 0.28 & $\mathrm{NA}$ & 0.60 & $0.26-1.39$ & 0.23 & NA & 1.75 & $0.78-3.94$ & 0.18 & NA & 0.97 & $0.64-1.48$ & 0.90 & 0 & 1.90 & $0.85-4.23$ & 0.12 & NA \\
\hline \multicolumn{22}{|c|}{ NOS evaluation } \\
\hline$\geq 7$ & 7 & 1.01 & $0.76-1.36$ & 0.92 & 74.2 & 1.04 & $0.80-1.36$ & 0.77 & 51.0 & 0.87 & $0.44-1.73$ & 0.70 & 60.3 & 1.03 & $0.75-1.41$ & 0.85 & 67.7 & 0.84 & $0.47-1.49$ & 0.55 & 46.9 \\
\hline$<7$ & 3 & 1.41 & $1.09-1.81$ & 0.01 & 0 & 0.97 & $0.47-2.00$ & 0.93 & 62.3 & 2.38 & $1.27-4.45$ & 0.01 & 16.9 & 1.21 & $0.92-1.59$ & 0.17 & 0 & 2.35 & $1.25-4.40$ & 0.01 & 0 \\
\hline
\end{tabular}




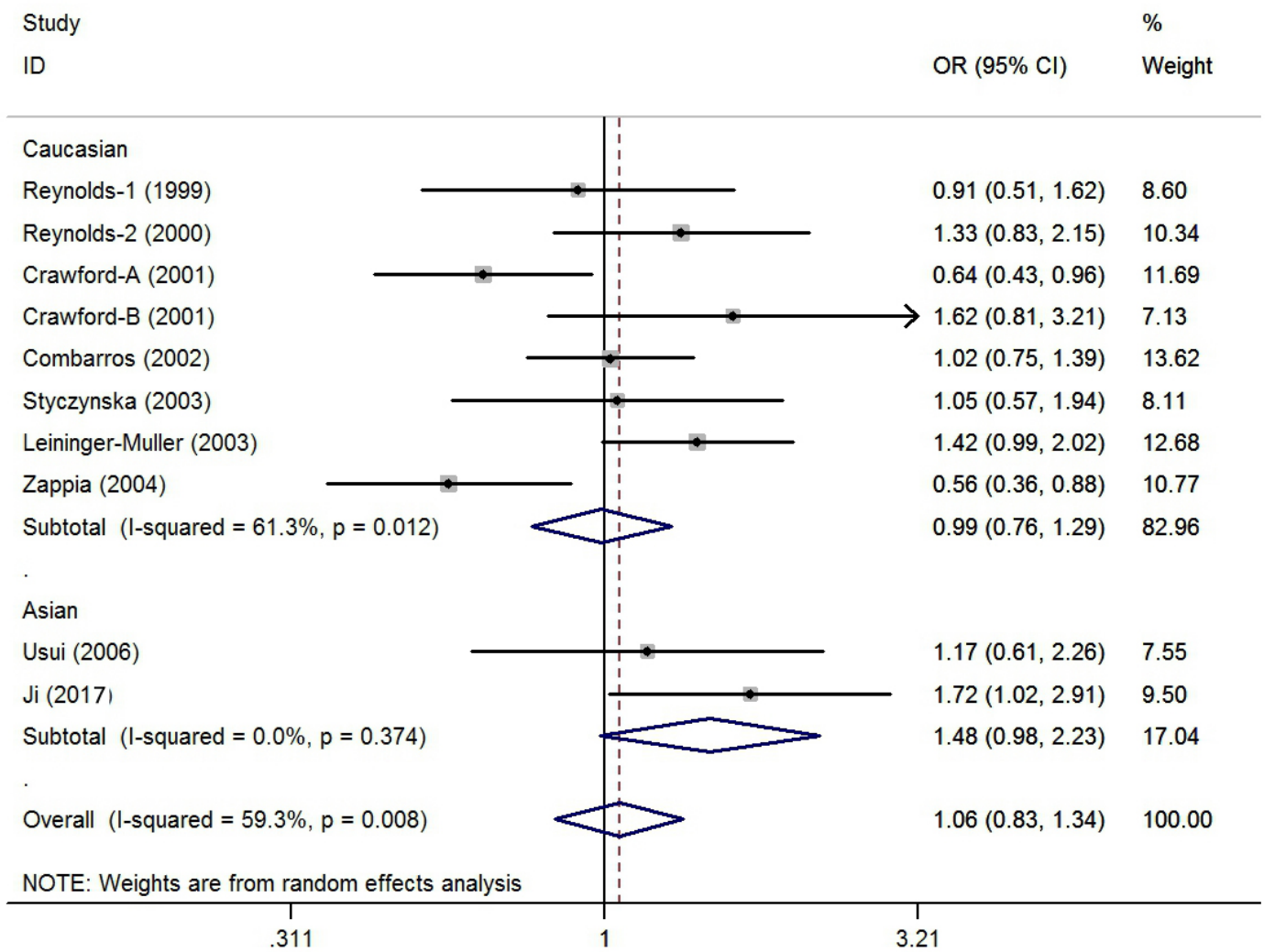

Fig. 2. OR and 95\% CIs of the associations between MPO rs2333227 G $>$ A polymorphism and AD risk in GA + AA vs. GG model.

difference, control design, and NOS evaluation differences between studies may contribute to heterogeneity. We performed subgroup analyses based on these factors. Heterogeneity was greatly reduced in the Asian group and the subgroup with a low NOS score but not in the Caucasian group or other subgroups (Table 2). Meta-regression analysis was conducted with the stratified factors, but the analysis did not identify any factor contributing to the heterogeneity (for GA + AA vs. GG model: $P_{H W \text { Estatus }}=$ 0.78, $P_{\text {Ethnicitydifference }}=0.26, P_{\text {Controldesign }}=0.95$, $P_{\text {NOSevaluation }}=0.71$; results were similar in other data not shown). Publication bias has been always recognized as a key factor influencing the synthesis of results from prior studies. Egger's linear regression test was conducted, and no asymmetry was observed in the funnel plots in the five genetic models. These funnel-plot results were confirmed by Egger's test (A vs. G: $P=0.37$; GA vs. GG: $P=0.93$; AA vs. GG: $P=$ 0.39; GA + AA vs. GG: $P=0.66$ (Fig. 3); AA vs. GG + GA: $P$ $=0.42$ ).

\subsection{Accumulative and sensitivity analyses}

Accumulative analyses (Fig. 4 for GA + AA vs. GG) and sensitivity analyses (Fig. 5 for GA + AA vs. GG) were conducted based on the published date of each study. No significant fluctuations were found in the results, which indicated that the results were stable and credible.

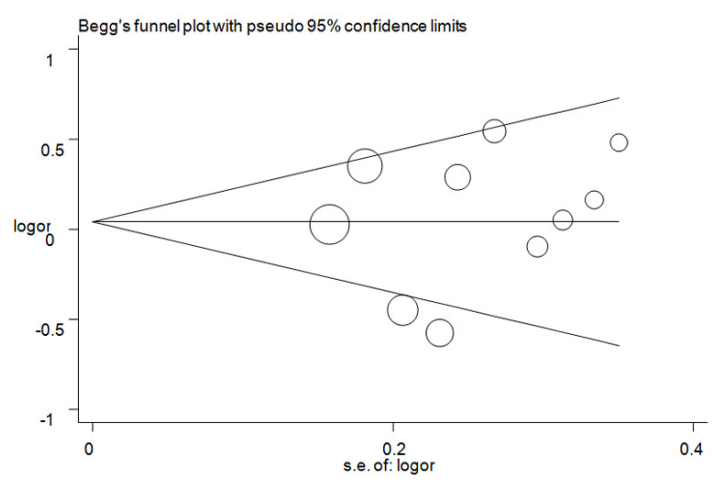

Fig. 3. Funnel plot analysis to detect publication bias for GA + AA vs. GG model of MPO rs2333227 G $>$ A polymorphism and AD risk. Circles represent the weight of the studies.

\subsection{Trial sequential analysis (TSA)}

TSA was conducted in the dominant model. An overall type I error rate of $5 \%$ and type II error rate of $20 \%$ were used. The total number of participants in this meta-analysis did not exceed the required information size of 11,452 (Fig. 6 for GA + AA vs. GG). The TSA indicated that the cumulative $\mathrm{z}$-curve neither crossed the trial monitoring boundary nor reached the required information size, indicating that the current evidence is insufficient and further studies are necessary. 
Study

ID

OR $(95 \% \mathrm{Cl})$

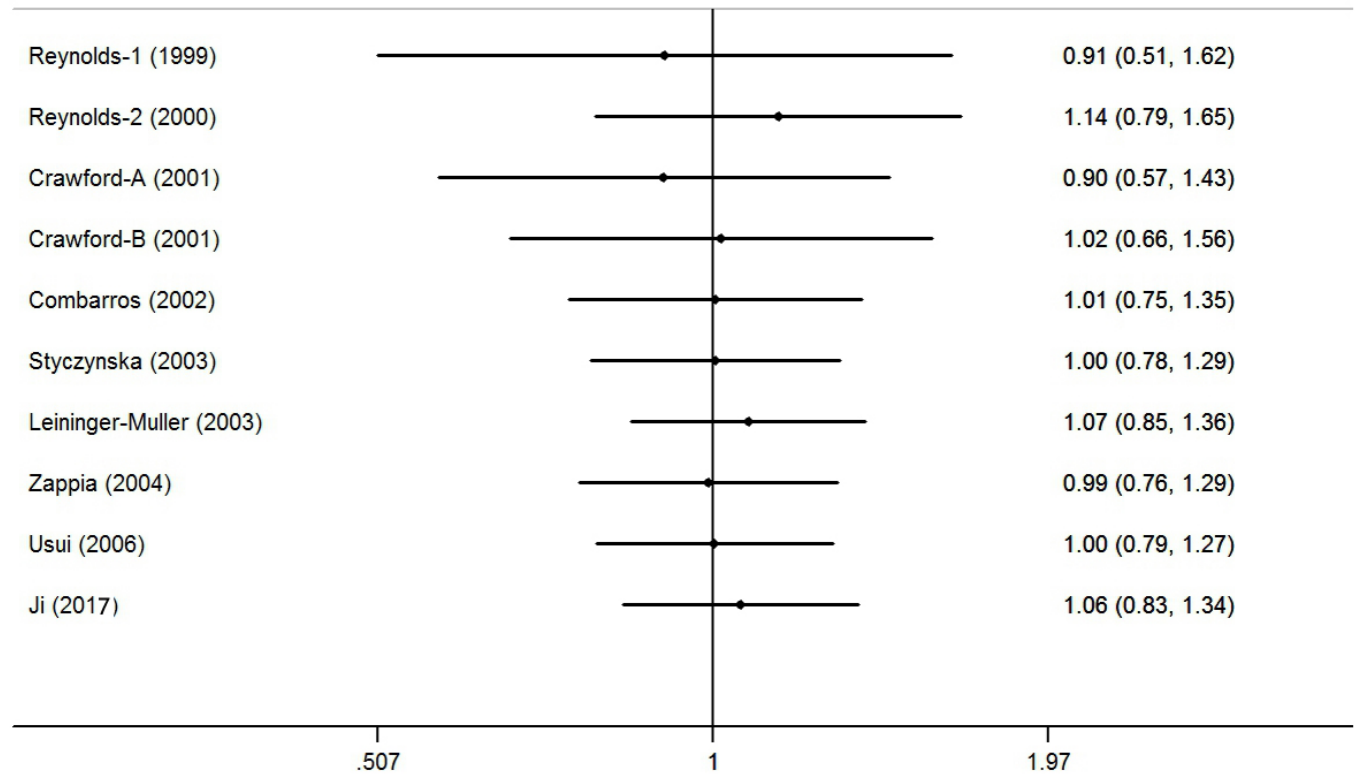

Fig. 4. Cumulative meta-analyses according to publication year in GA + AA vs. GG model of MPO rs2333227 G $>$ A polymorphism and AD risk.

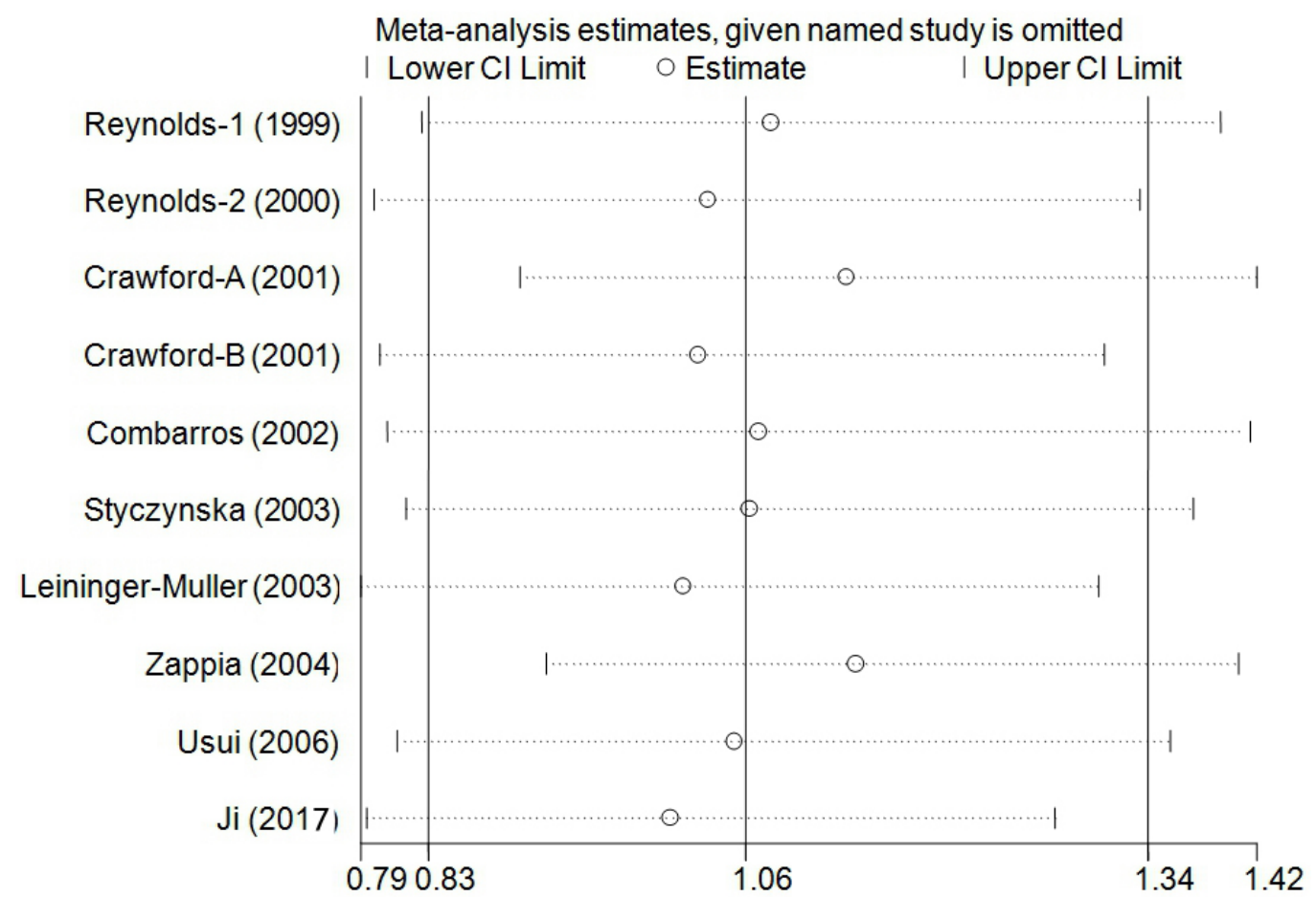

Fig. 5. Sensitivity analysis through deleting each study to reflect the influence of the individual dataset to the pooled ORs in GA + AA vs. GG model of MPO rs2333227 G $>$ A polymorphism and AD risk.

\section{Discussion}

$\mathrm{AD}$ is one of the most important neurodegenerative diseases and has been considered the most common cause of aging-associated dementia. The World Health Organization predicts that $\mathrm{AD}$ and other causes of dementia will overtake cancer and become the second leading cause of death [31]. The accumulation of plaques formed by $\mathrm{A} \beta$ peptide is the upstream causative factor that promotes neurodegeneration 


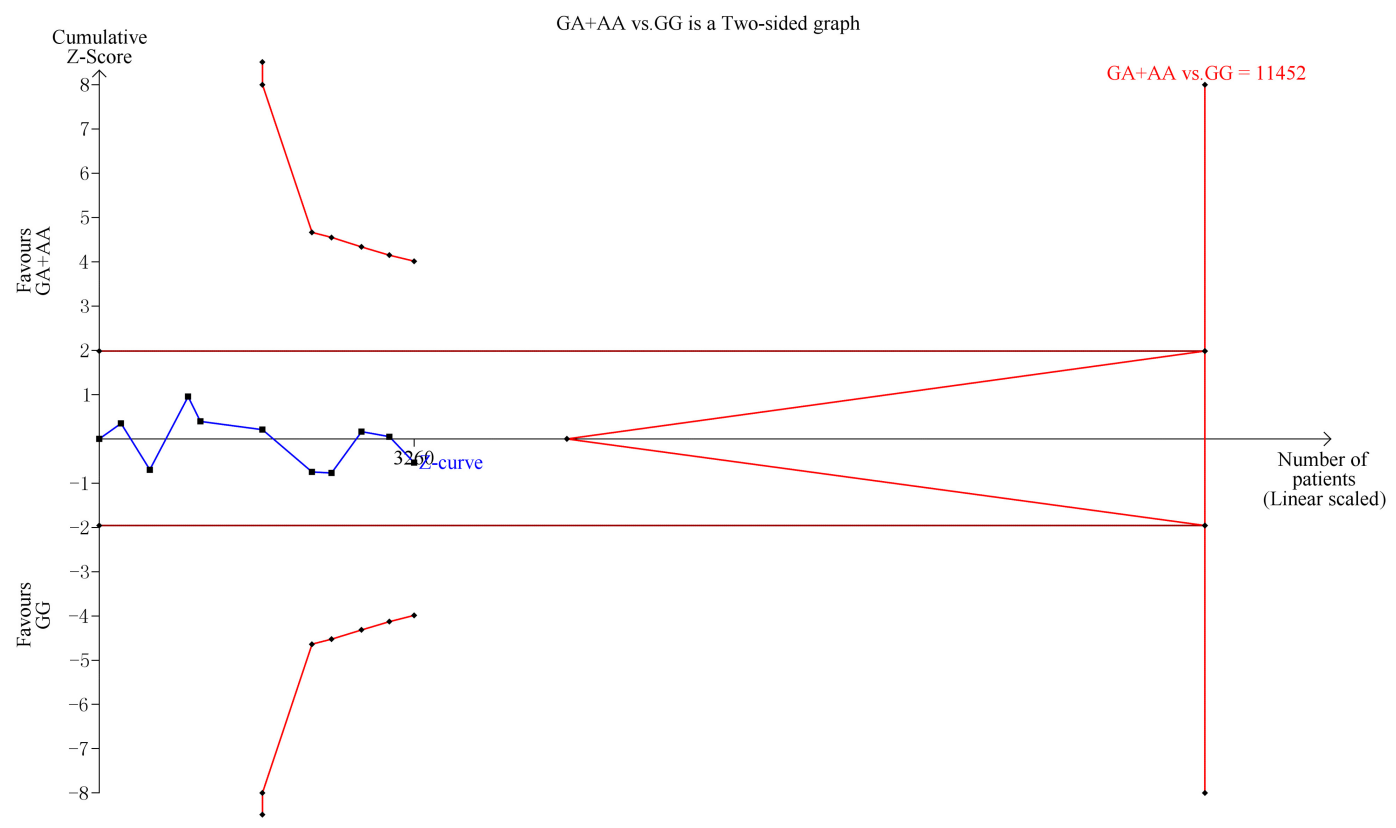

Fig. 6. Trial sequential analysis of MPO rs2333227 G $>$ A polymorphism and AD risk in GA + AA vs. GG model. The blue line represents the cumulative $\mathrm{Z}$-score of the meta-analysis. The red straight represent the conventional $P=0.05$ statistical boundaries.

$[32,33] . \mathrm{MPO}$ is an iron-containing heme protein, which is thought to be a hydrogen peroxide oxidoreductase and belongs to the mammalian family of peroxidases. Increasing evidence suggests a close relationship between MPO and its cytotoxic product, hypochlorous acid and AD. Animal research has also indicated that MPO expression and production promotes the stress-induced cross-linking of $\mathrm{A} \beta$ into insoluble beta sheet structures, thereby increasing the $\mathrm{A} \beta$ deposition in the brain [24, 34]. Whether MPO can be considered as a biomarker of $\mathrm{AD}$ is a concept for future investigations. In 2014, Tzikas et al. [14] conducted a case-control study on the plasma concentrations of MPO using enzyme linked immune absorbent assays and showed significantly higher plasma concentrations in AD patients than in comparison to elderly controls (AD vs. controls (mean $\pm \mathrm{SD}$ ): $132.8 \pm 114.8 \mathrm{ng} / \mathrm{mL}$ vs. $55.0 \pm 42.6 \mathrm{ng} / \mathrm{mL}$ ). Jin et al. [23] evaluated the plasma concentration of MPO in AD patients $(326.50 \pm 52.28 \mu \mathrm{g} / \mathrm{L})$ and healthy controls $(276.01 \pm 24.87 \mu \mathrm{g} / \mathrm{L})$ using an enzymelinked immunosorbent assay and found significantly lower concentrations of MPO in individuals with AA genotypes than individuals with GA and GG genotypes carriers in both groups.

In term of rs2333227 G>A polymorphism, some studies have suggested that this polymorphism influences MPO transcription level and strongly reduces mRNA expression. This may be due to this polymorphic site being located within an Alu hormone-responsive element, which may lead to the loss of a SP1 transcription factor binding site, and play a protective role by reducing MPO activity $[16,35,36]$. Subsequently, numerous studies have demonstrated that MPO rs2333227 G $>$ A polymorphism may influence susceptibilities to several diseases, such as different types of cancer [37-39], coronary artery disease [40], and diabetic nephropathy [41].

Firstly, Reynolds et al. [24] conducted acase-control study and reported that the MPO rs2333227 GG genotype increased the risk for $\mathrm{AD}$ in females in a US population in 1999. Since then, many studies that focused on the relationship between MPO rs2333227 G>A polymorphism and AD risk have been published. Similarly, Crawford et al. [26] also reported increased AD risk in MPO rs2333227 Gallelecarriers $(\mathrm{OR}=2.45,95 \% \mathrm{CI}=1.08-2.05)$ in the Caucasian population, but not in the Hispanic population. Moreover, Zappia et al. [29] reported a similar elevated risk for AD in individuals with the $\mathrm{GG}$ genotype $(\mathrm{OR}=1.78,95 \% \mathrm{CI}=1.13-2.80)$. In contrast, Leininger-Muller et al. [28] suggested that the MPO rs2333227 Aallelemight play a role in AD development. Ji et al. [23] also suggested that the MPO rs2333227 Aallele significantly increased the risk of $\mathrm{AD}$ in a Chinese population $(\mathrm{OR}=1.58,95 \% \mathrm{CI}=1.02-2.46)$. Moreover, Usui et al. [30] and others did not find any significant association between the MPO rs2333227 G > A polymorphism and AD risk $[18,27,30]$.

These discrepancies among published studies may have been derived from the following: (1) the study population came from different countries or ethnicities; (2) small sample size in the studies; (3) the quality of the included studies, which was inconsistent according to NOS evaluation; and (4) the controls of each study comprised population-based and hospital-based controls. So, our meta-analysis was therefore conducted to investigate and clarify the precise association. The pooled results, using general and stratified analyses based on the current evidence, indicated that no significant associ- 
ation exists between MPO rs2333227 G>A polymorphism and $\mathrm{AD}$ risk. Notably, the results of this meta-analysis need to be verified in additional studies using larger sample sizes, according to our TSA.

Compared with previous meta-analyses, five additional studies were enrolled in the present meta-analysis and a more comprehensive stratified analysis was conducted. In addition, cumulative and sensitivity analyses were conducted, as well as tests for publication bias, and the influence of NOS evaluation differences. Finally, a TSA was conducted to guarantee the stability and accuracy of our results. Furthermore, our meta-analysis had the following advantages: (1) a larger sample size contributing to more reliable results; (2) the use of a more scientific retrieval strategy and rigorous methodologies; (3) more subgroup analyses to explore potential relationships; (4) the use of TSA, which indicated that the current evidence is insufficient and further studies are necessary; and (5) no significant publication bias was identified that might have influenced the results.

Nevertheless, this meta-analysis had some limitations that should be addressed: (1) only nine studies could be found that focused on the association between rs2333227 G $>$ A polymorphism and AD susceptibility; (2) only the unadjusted assessment was included, without using controls measured in the original data, such as lifestyle, environmental exposure, and gene-environment interactions, which limited the exploration of interaction mechanisms; (3) published studies were all focused on Caucasian and Asian populations, which may have led to race bias; and (4) significant heterogeneity was found among the included studies, which was alleviated by subgroup analysis, suggesting that there may be potential factors that lead to heterogeneity, but these could not be identified using regression analysis due to the limited amount of currently available data.

\section{Conclusions}

In summary, the current meta-analysis suggested that the MPO rs2333227 G > A polymorphism is not a causal factor for AD. However, further case-control studies in various ethnicities, and using large sample sizes, are necessary to further validate our findings.

\section{Author contributions}

GLY and LZ designed the research study. ZCF and CCM performed the data curation. CCM, YJH analyzed the data. ZCF, CCM, YJH, GLY and LZ wrote the manuscript. All authors contributed to editorial changes in the manuscript.

\section{Ethics approval and consent to participate}

Not applicable.

\section{Acknowledgment \\ Not applicable.}

\section{Funding}

This study was supported by the Science and Technology Department of Hubei Province (No. 2020CFB747) and HuBei Provincial Department of Science and Technology Innovation Group Programme (No. 2019CFA034).

\section{Conflict of interest}

The authors declare no conflict of interest.

\section{References}

[1] Sommerlad A, Livingston G. Preventing Alzheimer's dementia. British Medical Journal. 2017; 359: j5667.

[2] Weuve J, Hebert LE, Scherr PA, Evans DA. Deaths in the United States among persons with Alzheimer's disease (20102050) Alzheimer's and Dementia. 2014; 10: e40-e46.

[3] Hebert LE, Weuve J, Scherr PA, Evans DA. Alzheimer disease in the United States (2010-2050) estimated using the 2010 census. Neurology. 2013; 80: 1778-1783.

[4] GBD 2016 Dementia Collaborators. Global, regional, and national burden of Alzheimer's disease and other dementias, 1990-2016: a systematic analysis for the Global Burden of Disease Study 2016. The Lancet Neurology. 2019; 18: 88-106.

[5] Karran E, Mercken M, De Strooper B. The amyloid cascade hypothesis for Alzheimer's disease: an appraisal for the development of therapeutics. Nature Reviews Drug Discovery. 2011; 10: 698712.

[6] Scheltens P, Blennow K, Breteler MMB, de Strooper B, Frisoni GB, Salloway S, et al. Alzheimer's disease. Lancet. 2016; 388: 505517.

[7] Bangen KJ, Nation DA, Delano-Wood L, Weissberger GH, Hansen LA, Galasko DR, et al. Aggregate effects of vascular risk factors on cerebrovascular changes in autopsy-confirmed Alzheimer's disease. Alzheimer's\& Dementia. 2015; 11: 394403.e1.

[8] Jiang T, Yu J, Tian Y, Tan L. Epidemiology and etiology of Alzheimer's disease: from genetic to non-genetic factors. Current Alzheimer Research. 2014; 10: 852-867.

[9] Genin E, Hannequin D, Wallon D, Sleegers K, Hiltunen M, Combarros O, et al. APOE and Alzheimer disease: a major gene with semi-dominant inheritance. Molecular Psychiatry. 2011; 16: 903907.

[10] Xia Q, Yang X, Shi J, Liu Z, Peng Y, Wang W, et al. The protective A673T mutation of Amyloid Precursor Protein (APP) in Alzheimer's Disease. Molecular Neurobiology. 2021; 58: 40384050.

[11] Prokopenko D, Morgan SL, Mullin K, Hofmann O, Chapman $\mathrm{B}$, Kirchner $\mathrm{R}$, et al. Whole-genome sequencing reveals new Alzheimer's disease-associated rare variants in loci related to synaptic function and neuronal development. Alzheimer's and Dementia. 2021.

[12] Lambert JC, Ibrahim-Verbaas CA, Harold D, Naj AC, Sims R, Bellenguez $\mathrm{C}$, et al. Meta-analysis of 74,046 individuals identifies 11 new susceptibility loci for Alzheimer's disease. Nature Genetics. 2013; 45: 1452-1458.

[13] Oyenuga AO, Couper D, Matsushita K, Boerwinkle E, Folsom AR. Association of monocyte myeloperoxidase with incident cardiovascular disease: the Atherosclerosis Risk in Communities Study. PLoSONE. 2018; 13: e0205310.

[14] Tzikas S, Schlak D, Sopova K, Gatsiou A, Stakos D, Stamatelopoulos $\mathrm{K}$, et al. Increased myeloperoxidase plasma levels in patients with Alzheimer's disease. Journal of Alzheimer's Disease. 2014; 39: 557-564.

[15] Morishita K, Tsuchiya M, Asano S, Kaziro Y, Nagata S. Chromosomal gene structure of human myeloperoxidase and regulation of its expression by granulocyte colony-stimulating factor. The Journal of Biological Chemistry. 1987; 262: 15208-15213. 
[16] Austin GE, Lam L, Zaki SR, Chan WC, Hodge T, Hou J, et al. Sequence comparison of putative regulatory DNA of the 5 ' flanking region of the myeloperoxidase gene in normal and leukemic bone marrow cells. Leukemia. 1993; 7: 1445-1450.

[17] Piedrafita FJ, Molander RB, Vansant G, Orlova EA, Pfahl M, Reynolds WF. An Alu element in the myeloperoxidase promoter contains a composite SP1-thyroid hormone-retinoic acid response element. The Journal of Biological Chemistry. 1996; 271: 1441214420.

[18] Combarros O, Infante J, Llorca J, Peña N, Fernández-Viadero C, Berciano J. The myeloperoxidase gene in Alzheimer's disease: a case-control study and meta-analysis. Neuroscience Letters. 2002; 326: 33-36.

[19] Stang A. Critical evaluation of the Newcastle-Ottawa scale for the assessment of the quality of nonrandomized studies in metaanalyses. European Journal of Epidemiology. 2010; 25: 603-605.

[20] Egger M, Davey Smith G, Schneider M, Minder C. Bias in metaanalysis detected by a simple, graphical test. British Medical Journal. 1997; 315: 629-634.

[21] Begg CB, Mazumdar M. Operating characteristics of a rank correlation test for publication bias. Biometrics. 1994; 50: 1088-1101.

[22] Wetterslev J, Thorlund K, Brok J, Gluud C. Trial sequential analysis may establish when firm evidence is reached in cumulative meta-analysis. Journal of Clinical Epidemiology. 2008; 61: 64-75.

[23] Ji W, Zhang Y. The association of MPO gene promoter polymorphisms with Alzheimer's disease risk in Chinese Han population. Oncotarget. 2017; 8: 107870-107876.

[24] Reynolds WF, Rhees J, Maciejewski D, Paladino T, Sieburg H, Maki RA, et al. Myeloperoxidase polymorphism is associated with gender specific risk for Alzheimer's disease. Experimental Neurology. 1999; 155: 31-41.

[25] Reynolds WF, Hiltunen M, Pirskanen M, Mannermaa A, Helisalmi S, Lehtovirta M, et al. MPO and APOEepsilon4 polymorphisms interact to increase risk for AD in Finnish males. Neurology. 2000; 55: 1284-1290.

[26] Crawford FC, Freeman MJ, Schinka JA, Morris MD, Abdullah LI, Richards D, et al. Association between Alzheimer's disease and a functional polymorphism in the Myeloperoxidase gene. Experimental Neurology. 2001; 167: 456-459.

[27] Styczynska M, Religa D, Pfeffer A, Luczywek E, Wasiak B, Styczynski G, et al. Simultaneous analysis of five genetic risk factors in Polish patients with Alzheimer's disease. Neuroscience Letters. 2003; 344: 99-102.

[28] Leininger-Muller B, Hoy A, Herbeth B, Pfister M, Serot JM, Stavljenic-Rukavina M, et al. Myeloperoxidase G-463a polymorphism and Alzheimer's disease in the ApoEurope study. Neuroscience Letters. 2003; 349: 95-98.

[29] Zappia M, Manna I, Serra P, Cittadella R, Andreoli V, La Russa A, et al. Increased risk for Alzheimer disease with the interaction of MPO and a2M polymorphisms. Archives of Neurology. 2004; 61: 341-344.

[30] Usui C, Shibata N, Ohnuma T, Higashi S, Ohkubo T, Ueki A, et al. No genetic association between the myeloperoxidase gene -463 polymorphism and estrogen receptor-alpha gene polymorphisms and Japanese sporadic Alzheimer's disease. Dementia and Geriatric Cognitive Disorders. 2006; 21: 296-299.

[31] Gammon K. Neurodegenerative disease: brain windfall. Nature. 2014; 515: 299-300.

[32] França MB, Lima KC, Eleutherio ECA. Oxidative stress and amyloid toxicity: insights from yeast. Journal of Cellular Biochemistry. 2017; 118: 1442-1452.

[33] Zheng L, Kågedal K, Dehvari N, Benedikz E, Cowburn R, Marcusson J, et al. Oxidative stress induces macroautophagy of amyloid beta-protein and ensuing apoptosis. Free Radical Biology and Medicine. 2009; 46: 422-429.

[34] Chen Y, Wang T, Rogers KA, Rutt BK, Ronald JA. Close association of myeloperoxidase-producing activated microglia with amyloid plaques in hypercholesterolemic rabbits. Journal of Alzheimer's Disease. 2019; 67: 1221-1234.

[35] Nikpoor B, Turecki G, Fournier C, Théroux P, Rouleau GA. A functional myeloperoxidase polymorphic variant is associated with coronary artery disease in French-Canadians. American Heart Journal. 2001; 142: 336-339.

[36] He C, Tamimi RM, Hankinson SE, Hunter DJ, Han J. A prospective study of genetic polymorphism in MPO, antioxidant status, and breast cancer risk. Breast Cancer Research and Treatment. 2009; 113: 585-594.

[37] Meng Q, Wu S, Wang Y, Xu J, Sun H, Lu R, et al. MPO promoter polymorphism rs2333227 enhances malignant phenotypes of colorectal cancer by altering the binding affinity of AP- $2 \alpha$. Cancer Research. 2018; 78: 2760-2769.

[38] Roszak A, Lutkowska A, Lianeri M, Sowińska A, Jagodziński PP. Involvement of myeloperoxidase gene polymorphism $463 \mathrm{G}>\mathrm{A}$ in development of cervical squamous cell carcinoma. The International Journal of Biological Markers. 2016; 31: e440-e445.

[39] Choi J, Neuhouser ML, Barnett MJ, Hong C, Kristal AR, Thornquist MD, et al. Iron intake, oxidative stress-related genes (MnSOD and MPO) and prostate cancer risk in CARET cohort. Carcinogenesis. 2008; 29: 964-970.

[40] Bushueva OY. Genetic Variants rs1049255 CYBA and rs2333227 MPO are Associated with susceptibility to coronary artery disease in Russian residents of central Russia. Kardiologiia. 2020; 60: 1229.

[41] Buraczynska K, Koziol-Montewka M, Majdan M, Tokarz A, Ksiazek A. Genetic determination of TNF and myeloperoxidase production in dialyzed patients with diabetic nephropathy. Renal Failure. 2004; 26: 633-639. 